Research Paper

\title{
High WNT6 expression indicates unfavorable survival outcome for patients with colorectal liver metastasis after liver resection
}

\author{
Jianhong Peng ${ }^{1 *}$, Yixin Zhao ${ }^{2 *}$, Qiuyun Luo ${ }^{3}$, Hao Chen 3 , Wenhua Fan ${ }^{1}$, Zhizhong Pan ${ }^{1}$, Xueping Wang ${ }^{3^{凶}}$, \\ Lin Zhang ${ }^{3 凶}$ \\ 1. Department of Colorectal Surgery, Sun Yat-sen University Cancer Center; State Key Laboratory of Oncology in South China; Collaborative Innovation \\ Center for Cancer Medicine, Guangzhou, Guangdong 510060, P. R. China. \\ 2. Department of Anesthesiology \& Operating Theatre, Sun Yat-sen University Cancer Center; State Key Laboratory of Oncology in South China; \\ Collaborative Innovation Center for Cancer Medicine, Guangzhou, Guangdong 510060, P. R. China. \\ 3. Department of Clinical Laboratory, Sun Yat-sen University Cancer Center; State Key Laboratory of Oncology in South China; Collaborative Innovation \\ Center for Cancer Medicine, Guangzhou, Guangdong 510060, P. R. China. \\ * Jianhong Peng and Yixin Zhao contributed equally to this work.
}

$\triangle$ Corresponding authors: Xueping Wang and Lin Zhang, Department of Clinical Laboratory, Sun Yat-sen University Cancer Center, 651 Dongfeng Road East, Guangzhou, Guangdong 510060, P. R. China. Tel: +86-20-87343124; Fax: +86-20-87343637; E-mails: zhanglin@sysucc.org.cn

(c) Ivyspring International Publisher. This is an open access article distributed under the terms of the Creative Commons Attribution (CC BY-NC) license (https://creativecommons.org/licenses/by-nc/4.0/). See http://ivyspring.com/terms for full terms and conditions.

Received: 2019.01.04; Accepted: 2019.05.07; Published: 2019.06.02

\begin{abstract}
Objective: As a member of the Wnt family, WNT6 contributes to tumorigenesis and the development of various types of cancer. However, the expression status of WNT6 in colorectal liver metastasis (CRLM) and its prognostic value remain to be elucidated. In this study, we evaluated the association of WNT6 expression with survival outcomes in CRLM patients undergoing liver resection.

Methods: The medical records of 106 consecutive CRLM patients undergoing curative tumor resection between October 1996 and December 2011 were retrospectively selected. WNT6 expression was detected using immunohistochemistry (IHC) analyses on paraffin-embedded specimens. The IHC score was determined according to the percentage and intensity of positively stained cells. Recurrence-free survival (RFS) and overall survival (OS) were analyzed using the Kaplan-Meier method and the log-rank test, and independent prognostic factors were determined by Cox regression modeling.

Results: We found that WNT6 was commonly expressed in $93.4 \%$ (99/106) of colorectal cancer tissues. The median IHC score of WNT6 expression was significantly lower in patients receiving preoperative chemotherapy than those without preoperative chemotherapy $(1.33 \mathrm{vs} .2 .33, P=0.033)$. Survival analysis indicated that patients with high WNT6 expression had poorer 5-year OS than those with low WNT6 expression ( $31.0 \%$ vs. $62.2 \%, P=0.012)$. The 5 -year OS rate was significantly lower in the high WNT6 group than in the low WNT6 group $(36.8 \%$ vs. $79.9 \%, P=0.013)$ in low-risk patients but was comparable among the high-risk patients $(22.7 \%$ vs. $34.7 \%, P=0.433)$. Multivariate analysis indicated that high WNT6 expression was independently associated with poor OS (hazard ratio [HR]: $2.089 ; 95 \%$ confidence interval [Cl]: 1.231-3.545; $\mathrm{P}=0.006)$.

Conclusions: High expression of WNT6 was associated with unfavorable oncologic prognosis in patients with CRLM undergoing liver resection. Detection of WNT6 expression may be valuable for guiding postoperative treatment.
\end{abstract}

Key words: WNT6, colorectal liver metastasis, expression, survival

\section{Introduction}

To date, colorectal cancer (CRC) has become one of the most common human malignant diseases and a leading cause of cancer-related deaths in China and worldwide [1, 2]. The survival outcome of CRC 
patients is largely impaired by the development of distant metastatic disease. The liver, in addition to being the most common site of metastases, is also the first area of metastatic spread in $25 \%$ of patients at diagnosis [3]. In addition, approximately $25-35 \%$ of patients ultimately develop liver metastases during the course of their disease [4]. Despite recent advances in chemotherapy strategies for the treatment of patients with colorectal liver metastases (CRLM), liver resection offers the only chance of long-term survival for these patients [5-7]. Unfortunately, more than $60 \%$ of patients developed recurrent disease after initial liver resection during follow-up [8,9]. Among those with postoperative recurrence, $27.8-45 \%$ of patients even developed early recurrent disease within 6 to 8 months after liver resection $[10,11]$. Therefore, the management of CRLM is challenging, and understanding the tumor biological behavior of CRLM is important to help establish and optimize therapeutic strategies. Although multiple clinical risk factors are commonly adopted in the identification of different risk subgroups, a proportion of patients remain incorrectly assessed because of the inconsistent predictive power and lack of reproducibility due to selection bias $[12,13]$. Identification of novel biomarkers to screen out various prognostic risk subgroups to guide individual treatment for CRLM is urgently needed.

Wnt/ $\beta$-catenin alterations are prominent in human malignancies and participate in tumorigenesis and tumor progression by increasing tumorigenicity, sustaining proliferation, and enhancing metastatic potential $[14,15]$. The Wnt gene family consists of structurally related genes that encode a group of 19 secreted and cysteine-rich signaling proteins [16]. Wnt family member 6 (WNT6), as a member of the Wnt signaling family, has been reported to be involved in promoting the proliferation and differentiation of embryonic palatal mesenchymal cells, macrophages, and stromal cells [17-19]. In addition to its vital functions in embryonic development, WNT6 also contributes to tumorigenesis [20]. Furthermore, previous studies have reported that WNT6 is overexpressed in gastric cancer and esophageal cancer, which indicates unfavorable survival after tumor resection [21, 22]. However, the prognostic value of WNT6 expression in patients with CRLM undergoing liver resection remains unclear. Overexpression of WNT6 is involved in colon cancer cell progression and promotes proliferation, cell cycle and migration [23]. Accordingly, we hypothesized that WNT6 is a direct measure of tumor biology and may be a powerful predictor of survival outcome in CRC patients.
To further validate our hypothesis, the present study aimed to explore the prognostic value of WNT6 and its relationship with clinicopathologic characteristics in patients with colorectal liver-only metastasis (CLOM) undergoing liver resection.

\section{Materials and methods}

\section{Patient selection}

Paraffin-embedded tumor tissue samples of primary tumors were obtained from 106 consecutive CRLM patients who underwent tumor resection at the Sun Yat-sen University Cancer Center (Guangzhou, China) from October 1996 to December 2011. All included patients met the following inclusion criteria: (1) histologically confirmed colorectal adenocarcinoma; (2) American Society of Anesthesiologists (ASA) class I-II status; (3) no preoperative extrahepatic metastases; (4) radical resection for both colorectal primary tumor and liver metastasis; and (5) a postoperative follow-up period of at least 3 months. The patient demographics, tumor characteristics, adjuvant chemotherapy and follow-up data were retrospectively collected from the electronic medical record system. The tumor stage was classified according to the 2010 American Joint Committee on Cancer staging system. The treatment strategy and operability of liver metastases for each patient were determined according to the final agreement of the multidisciplinary team (MDT) as previously described [24]. The present study was undertaken in accordance with the ethical standards of the World Medical Association Declaration of Helsinki. Informed consent for the use of tissue samples was obtained from the patients before tumor resection. The study was approved by the Institutional Research Ethics Committee of Sun Yat-sen University Cancer Center (Approval number: GZR2017-004).

\section{Immunohistochemistry}

The paraffin-embedded tissues were sectioned continuously into $4-\mu \mathrm{m}$-thick sections. The tissue sections were dewaxed in xylene, rehydrated and rinsed in graded ethanol solutions. The antigens were retrieved by heating the tissue sections at $100{ }^{\circ} \mathrm{C}$ for 5 min in EDTA (1 mmol/L, $\mathrm{pH} 8.0)$ solution when necessary. The sections were then immersed in a $0.3 \%$ hydrogen peroxide solution for $10 \mathrm{~min}$ to block endogenous peroxidase activity, rinsed in phosphate buffered saline (PBS) for $5 \mathrm{~min}$, and incubated with the WNT6 primary antibody (1:200 dilution, ab50030; Abcam, Cambridge, MA, USA) at $4{ }^{\circ} \mathrm{C}$ overnight. Subsequently, the slides were washed with $1 \times$ PBS and treated with a goat antibody against a mouse/rabbit secondary antibody (Envision; Dako, Glostrup, Denmark) at $37.5^{\circ} \mathrm{C}$ for $30 \mathrm{~min}$. Finally, the 
visualized staining was developed with 3,3'-diaminobenzidine tetrahydrochloride (DAB, Dako, Glostrup, Denmark), and all of the slides were counterstained with hematoxylin.

\section{Immunohistochemical scoring}

The immunohistochemistry (IHC) score of WNT6 expression was determined by the semiquantitative method according to the percentage and intensity of positively stained cells as we previously described [25, 26]. The positive staining was scored as follows: "0" (less than $5 \%$ positively stained cells), "1" (5-24\% positively stained cells), "2" (25-49\% positively stained cells), “3” (50-74\% positively stained cells), and " 4 " (75-100\% positively stained cells). The intensity was scored as follows: " 0 " (negative staining); "1" (weak staining); "2" (moderate staining); and " 3 " (strong staining). The final score was generated by multiplying the percentage score by the staining intensity score. Two independent observers blindly evaluated the IHC score of WNT6 expression in all specimens. The cut-off value of WNT6 expression was determined by the median IHC score. High WNT6 expression grade was defined when the IHC score was greater than the cut-off value.

\section{Recurrence risk assessment}

Recurrence risk in all CRLM patients after liver resection was evaluated by Fong clinical risk score (CRS) system [27]. The scoring system consisted of 5 clinical factors: (1) node-positive primary tumor, (2) largest metastasis $>5 \mathrm{~cm}$, (3) multiple liver metastases, (4) preoperative carcinoembryonic antigen (CEA) level > $200 \mathrm{ng} / \mathrm{mL}$, and (5) disease-free interval from primary tumor resection to the diagnosis of liver metastasis $<12$ months. Based on the number of risk factors, each patient was allocated a CRS. Accordingly, patients with a CRS of 0-2 were identified as the low-risk subgroup, while patients with a CRS of 3-5 were identified as the high-risk subgroup.

\section{Follow-up}

Patients were monitored through subsequent visits every 3 months for the first 2 years and then semiannually for 5 years after liver resection. Clinical examination, assessment of CEA and carbohydrate antigen 19-9 (CA19-9) levels, and chest radiography were conducted every 3 months. Chest/abdominal/ pelvic CT and colonoscopy were performed annually. Overall survival (OS) was defined as the interval from the date of liver resection to the date of death from any cause or to the last follow-up. Recurrence-free survival (RFS) was defined as the interval from tumor resection to the date of disease recurrence, death or the last follow-up. Random censoring was applied to patients without recurrence or death at the last follow-up date. The final follow-up visit occurred in May 2018.

\section{Statistical analysis}

All analyses were performed using IBM SPSS statistics software, version 21.0 (IBM Corp., Armonk, NY, USA). Categorical variables are presented as percentages, and they were compared using the chi-square test or Fisher's exact test. Continuous variables are presented as the median (range). Mann-Whiney $U$ test was used for two-group comparison, and Kruskal-Wallis $\mathrm{H}$ test was used for multiple-group comparison. Survival outcomes were summarized by the Kaplan-Meier method and log-rank survival analysis. Parameters for which $\mathrm{P}<$ 0.05 for OS in the univariate Cox models were further assessed in multivariate Cox models. Hazard ratios (HRs) and 95\% confidence intervals (CIs) were subsequently calculated. All of the statistical tests were two-sided; $\mathrm{P}<0.05$ was considered significant.

\section{Results}

\section{Patient characteristics}

A total of 106 eligible patients with CRLM were included in the present study. The clinical information is summarized in detail in Table 1. The mean age at diagnosis was 58 years (range, 25-77 years), with 69 $(65.1 \%)$ males and $37(34.9 \%)$ females. The median number of liver metastases in patients was 1 (range 1-11). With regard to CRS, 63 (49.4\%) patients were classified into the low-risk group (CRS 0-2), while 63 $(50.6 \%)$ patients were classified into the high-risk group (CRS 3-5). Regarding perioperative treatment, $31(29.2 \%)$ patients received preoperative chemotherapy, 94 (88.7\%) patients received adjuvant chemotherapy, and $11(10.4 \%)$ patients received intraoperative radiofrequency ablation.

\section{Association of WNT6 expression with clinicopathologic characteristics}

As shown in Figure 1, positive staining of WNT6 was mainly located in the cytoplasm of the cells, which was observed in 93.4\% (99/106) of colorectal cancer tissues. As presented in Figure 2, the cut-off value in WNT6 expression was the median IHC score of 2. Accordingly, a high expression level of WNT6 in tumor cells was noted in samples from 52 (49.1\%) patients. Subsequently, we assessed the association of WNT6 expression in tumor tissues with the following clinicopathologic parameters: age, sex, tumor location, tumor size, primary tumor differentiation, $\mathrm{T}$ stage, $\mathrm{N}$ stage, number and size of liver metastatic tumors, preoperative CEA and CA19-9 levels, perioperative chemotherapy, and survival outcome. 
The associations between clinicopathological features and WNT6 expression in tumor cells from 106 CRLM patients are summarized in Table 2. High WNT6 expression was associated with a higher mortality rate after liver resection than low WNT6 expression $(65.5 \%$ vs. $46.3 \%, \mathrm{P}=0.048)$. No significant association was found between WNT6 expression and other clinicopathological characteristics. The median IHC scores of WNT6 expression in CRS 1, CRS 2, CRS 3 and CRS 4 were 1.67 (range 0.67-8.00), 2.00 (range $0.00-12.00$ ), 2.33 (range 0.00-11.00) and 2.00 (range $0.67-7.33)$, respectively, which were not significantly different $(\mathrm{P}=0.995$, Figure $3 \mathrm{~A})$. However, the median IHC score of WNT6 expression in patients receiving preoperative chemotherapy was significantly lower than the score of those without preoperative chemotherapy $[1.33$ (range $0.00-7.33$ ) vs. 2.33 (range $0.00-12.00), \mathrm{P}=0.033$, Figure 3B].

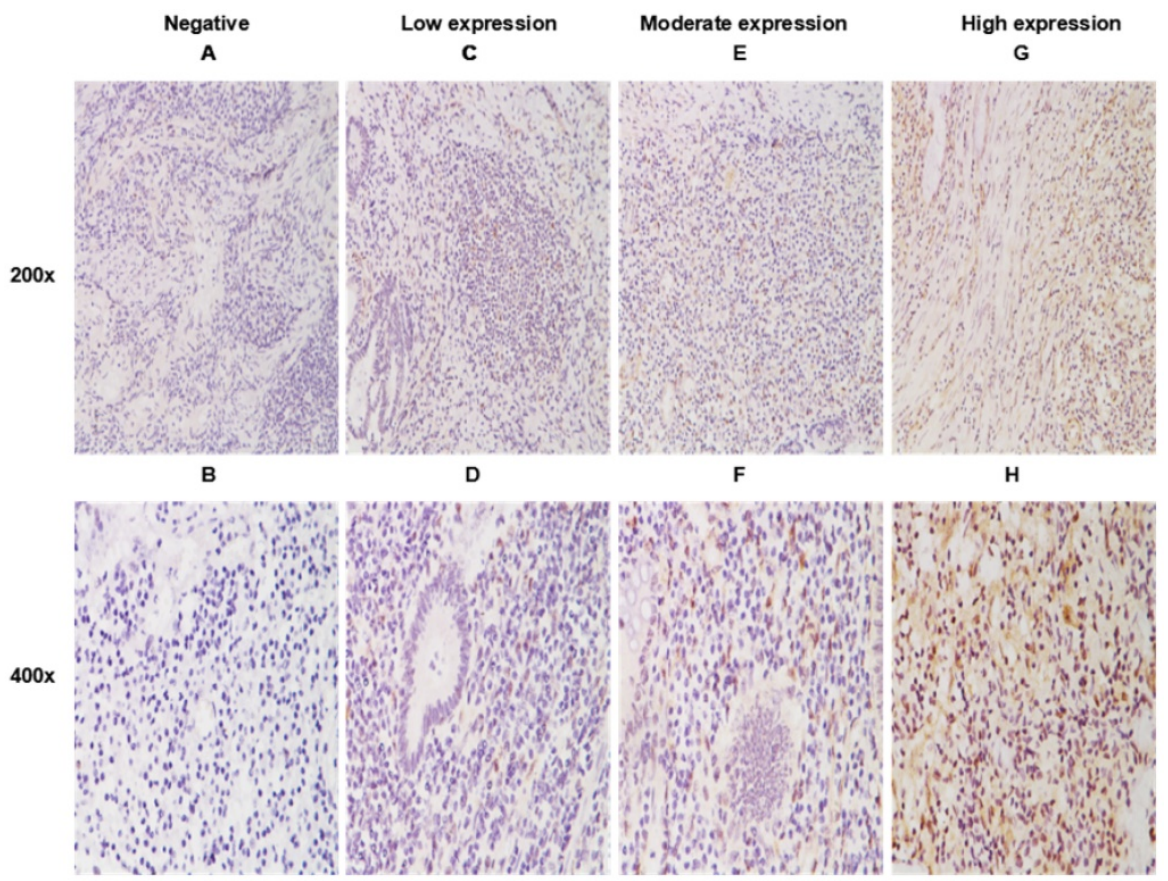

Figure 1. WNT6 expression in primary tumor tissues by immunohistochemistry (IHC). A. Negative expression of WNT6 protein (200x). C. Weak expression of WNT6 protein (200x). E. Moderate expression of WNT6 protein (200x). G. Intense expression of WNT6 protein (200x). B, D, F and H demonstrate a higher magnification (400x) of the area of the box in A, C, E and G, respectively.

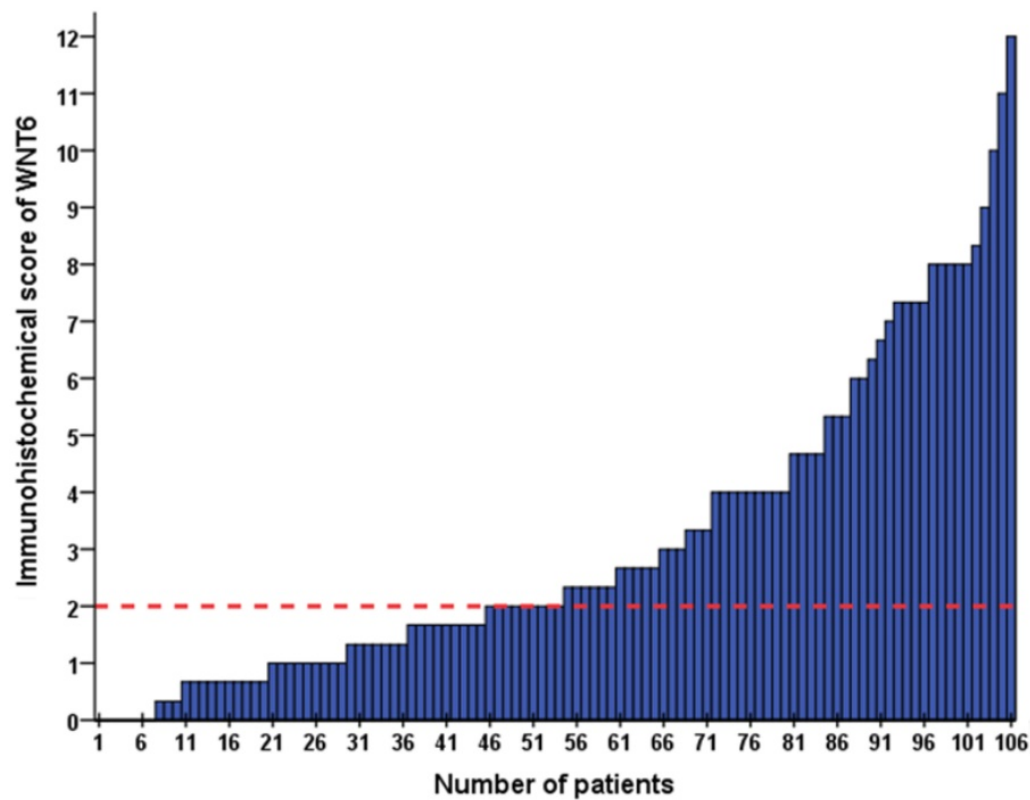

Figure 2. The IHC score of WNT6 expression in 106 patients with colorectal liver metastasis (CRLM). The red dotted line indicates the cut-off value in WNT6 expression as the median IHC score of 2. 
Table 1. Clinical data of 106 cases of colorectal liver metastasis patients

\begin{tabular}{|c|c|}
\hline Parameters & Total patients $(\mathrm{n}, \%)$ \\
\hline \multicolumn{2}{|c|}{ Patient characteristics } \\
\hline Median age (year) & $58(25-77)$ \\
\hline \multicolumn{2}{|l|}{ Age, year } \\
\hline$\leq 60$ & $62(58.5)$ \\
\hline$>60$ & $44(41.5)$ \\
\hline \multicolumn{2}{|l|}{ Sex } \\
\hline Male & $69(65.1)$ \\
\hline Female & $37(34.9)$ \\
\hline \multicolumn{2}{|c|}{ Primary tumor location } \\
\hline Right-side colon & $28(26.4)$ \\
\hline Left-side colon & $38(35.8)$ \\
\hline Rectum & $40(37.7)$ \\
\hline \multicolumn{2}{|c|}{ Primary tumor differentiation } \\
\hline Well & $2(1.9)$ \\
\hline Moderate & $89(84.0)$ \\
\hline Poor & $15(14.1)$ \\
\hline \multicolumn{2}{|l|}{ T stage } \\
\hline 2 & $8(7.5)$ \\
\hline 3 & $36(34.0)$ \\
\hline 4 & $62(58.5)$ \\
\hline \multicolumn{2}{|l|}{$\mathrm{N}$ stage } \\
\hline 0 & $33(31.1)$ \\
\hline 1 & $39(36.8)$ \\
\hline 2 & $34(32.1)$ \\
\hline \multicolumn{2}{|c|}{ Timing of liver metastasis } \\
\hline Synchronous & $99(93.4)$ \\
\hline Metachronous & $7(6.6)$ \\
\hline \multicolumn{2}{|c|}{ Number of liver metastatic tumors } \\
\hline 1 & $56(52.8)$ \\
\hline 2 & $23(21.7)$ \\
\hline 3 & $8(7.5)$ \\
\hline 4 & $4(3.8)$ \\
\hline 5 & $2(1.9)$ \\
\hline$>5$ & $13(12.3)$ \\
\hline \multicolumn{2}{|c|}{ Metastases diameter $(\mathrm{cm})$} \\
\hline Median (range) & $2.5(0.5-12)$ \\
\hline$\leq 3$ & $67(63.2)$ \\
\hline$>3$ & $39(36.8)$ \\
\hline \multicolumn{2}{|c|}{ Preoperative CEA (ng/ml) } \\
\hline$\leq 10$ & $49(46.2)$ \\
\hline$>10$ & $57(53.8)$ \\
\hline \multicolumn{2}{|c|}{ Preoperative CA19-9 (U/ml) } \\
\hline$\leq 35$ & $74(69.8)$ \\
\hline$>35$ & $32(30.2)$ \\
\hline \multicolumn{2}{|l|}{ Clinical risk score } \\
\hline 1 & $17(16.0)$ \\
\hline 2 & $46(43.4)$ \\
\hline 3 & $31(29.2)$ \\
\hline 4 & $12(11.3)$ \\
\hline \multicolumn{2}{|c|}{ Intraoperative radiofrequency ablation } \\
\hline Yes & $11(10.4)$ \\
\hline No & $95(89.6)$ \\
\hline \multicolumn{2}{|c|}{ Preoperative chemotherapy } \\
\hline Yes & $31(29.2)$ \\
\hline No & $75(70.8)$ \\
\hline \multicolumn{2}{|c|}{ Adjuvant chemotherapy } \\
\hline Yes & $12(11.3)$ \\
\hline No & $94(88.7)$ \\
\hline
\end{tabular}

Table 2. Association of the expression of WNT6 and clinicopathologic parameters in all patients

\begin{tabular}{llll}
\hline Parameters & $\begin{array}{l}\text { Low WNT6 expression } \\
(\mathbf{n = 5 4}, \mathbf{0})\end{array}$ & $\begin{array}{l}\text { High WNT6 expression } \\
(\mathbf{n}=\mathbf{5 2}, \mathbf{0})\end{array}$ & P value \\
\hline Age, year & & & \\
$\leq 60$ & $35(64.8)$ & $27(51.9)$ & 0.178 \\
$>60$ & $19(35.2)$ & $25(48.1)$ & \\
Sex & & & \\
Male & $37(68.5)$ & $32(61.5)$ & 0.451 \\
\hline
\end{tabular}

\begin{tabular}{|c|c|c|c|}
\hline Parameters & $\begin{array}{l}\text { Low WNT6 expression } \\
(\mathrm{n}=54, \%)\end{array}$ & $\begin{array}{l}\text { High WNT6 expression } \\
(n=52, \%)\end{array}$ & Pvalue \\
\hline Female & $17(31.5)$ & $20(38.5)$ & \\
\hline \multicolumn{4}{|l|}{$\begin{array}{l}\text { Primary tumor } \\
\text { location }\end{array}$} \\
\hline Right-side colon & $15(27.8)$ & $13(25.0)$ & 0.856 \\
\hline Left-side colon & $20(37.0)$ & $18(34.6)$ & \\
\hline Rectum & $19(35.2)$ & $21(40.4)$ & \\
\hline \multicolumn{4}{|l|}{$\begin{array}{l}\text { Primary tumor } \\
\text { differentiation }\end{array}$} \\
\hline Well to moderate & $46(85.2)$ & $45(86.5)$ & 0.842 \\
\hline Poor & $8(14.8)$ & $7(13.5)$ & \\
\hline \multicolumn{4}{|l|}{ T stage } \\
\hline $1-3$ & $21(38.9)$ & $22(42.3)$ & 0.720 \\
\hline 4 & $33(61.1)$ & $30(57.7)$ & \\
\hline \multicolumn{4}{|l|}{ N stage } \\
\hline 0 & $16(29.6)$ & $17(32.7)$ & 0.734 \\
\hline $1-2$ & $38(70.4)$ & $35(67.3)$ & \\
\hline \multicolumn{4}{|c|}{ Number of liver metastatic tumors } \\
\hline $1-3$ & $45(83.3)$ & $42(80.8)$ & 0.731 \\
\hline$>3$ & $9(16.7)$ & $10(19.2)$ & \\
\hline \multicolumn{4}{|l|}{$\begin{array}{l}\text { Metastases } \\
\text { diameter }(\mathrm{cm})\end{array}$} \\
\hline$\leq 3$ & $37(68.5)$ & $30(57.7)$ & 0.248 \\
\hline$>3$ & $17(31.5)$ & $22(42.3)$ & \\
\hline \multicolumn{4}{|c|}{$\begin{array}{l}\text { Preoperative CEA } \\
(\mathrm{ng} / \mathrm{ml})\end{array}$} \\
\hline$\leq 10$ & $26(48.1)$ & $23(44.2)$ & 0.686 \\
\hline$>10$ & $28(51.9)$ & $29(55.8)$ & \\
\hline \multicolumn{4}{|l|}{ Preoperative } \\
\hline \multicolumn{4}{|l|}{ CA19-9 (U/ml) } \\
\hline$\leq 35$ & $40(74.1)$ & $34(65.4)$ & 0.330 \\
\hline$>35$ & $14(25.9)$ & $18(34.6)$ & \\
\hline $\begin{array}{l}\text { Intraoperative } \\
\text { radiofrequency } \\
\text { ablation }\end{array}$ & & & 0.307 \\
\hline Yes & $4(7.4)$ & $7(13.5)$ & \\
\hline No & $50(92.6)$ & $45(86.5)$ & \\
\hline \multicolumn{4}{|l|}{$\begin{array}{l}\text { Preoperative } \\
\text { chemotherapy }\end{array}$} \\
\hline Yes & $34(63.0)$ & $41(78.8)$ & 0.072 \\
\hline No & $20(37.0)$ & $11(21.2)$ & \\
\hline \multicolumn{4}{|l|}{$\begin{array}{l}\text { Adjuvant } \\
\text { chemotherapy }\end{array}$} \\
\hline Yes & $48(88.9)$ & $46(88.5)$ & 0.945 \\
\hline No & $6(11.1)$ & $6(11.5)$ & \\
\hline \multicolumn{4}{|l|}{$\begin{array}{l}\text { Postoperative } \\
\text { recurrence }\end{array}$} \\
\hline Yes & $34(63.0)$ & 38 (73.1) & 0.265 \\
\hline No & $20(37.0)$ & $14(26.9)$ & \\
\hline \multicolumn{4}{|l|}{ Survival status } \\
\hline Alive & $29(53.7)$ & $18(34.6)$ & 0.048 \\
\hline Dead & $25(46.3)$ & $34(65.5)$ & \\
\hline
\end{tabular}

\section{Association between WNT6 expression and survival outcome}

After liver resections, all patients were followed up for a median of 35.5 months (range: 2.0-143.3 months). Overall, $72(67.9 \%)$ patients experienced tumor recurrence, including $45.8 \%$ (33/72) patients with intrahepatic recurrence, $6.9 \%(5 / 72)$ patients with lung metastases, 5.6\% (4/72) patients with abdominal pelvic metastases, $11.8 \%(10 / 106)$ patients with multiple organ metastases, and $27.8 \%(20 / 72)$ patients with other metastases. As a result, 59 (55.7\%) patients died of tumor progression. Regarding the total patients, the 3- and 5-year RFS rates were 33.9\% and $30.6 \%$, respectively, and the 3 - and 5-year OS 
rates were $54.9 \%$ and $47.0 \%$, respectively. The 5 -year RFS did not significantly differ between the high and low WNT6 groups $(24.9 \%$ vs. $36.2 \%, \mathrm{P}=0.205$, Figure $4 \mathrm{~A})$. However, the 5 -year OS rates were significantly lower in the high WNT6 group than in the low WNT6 group $(31.0 \%$ vs. $62.2 \%, \mathrm{P}=0.012$, Figure $4 \mathrm{~B})$. The patients were further stratified as low risk for recurrence (CRS 1-2) or high risk (CRS 3-4). Among the low-risk patients, the 5-year OS rate was significantly lower in the high WNT6 group than in the low WNT6 group (36.8\% vs. $79.9 \%, \mathrm{P}=0.013$, Figure 5A). Among the high-risk patients, the 5-year OS rate was comparable between the high and low WNT6 groups ( $22.7 \%$ vs. $34.7 \%, P=0.433$, Figure $5 \mathrm{~B})$.

As shown in Table 3, the univariate analysis revealed that high WNT6 expression (HR: 1.920; 95\%

A

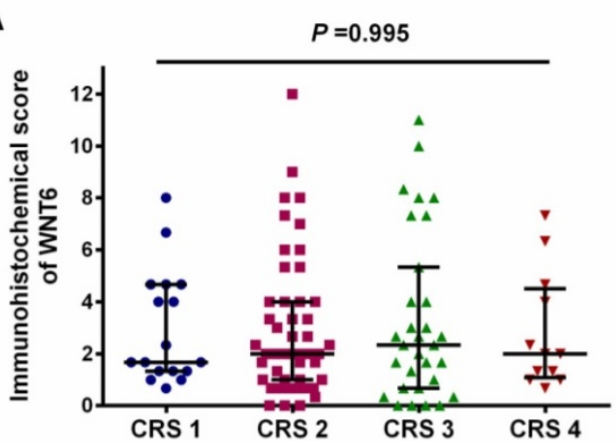

CI: 1.143-3.225; $\mathrm{P}=0.014), \mathrm{N}$ stage 1-2 (HR: 1.922; 95\% CI: 1.034-3.572; $\mathrm{P}=0.039)$, more than 3 liver metastases (HR: 2.481; 95\% CI: 1.320-4.663; P = 0.005), high preoperative CA19-9 (HR: 2.194; 95\% CI: 1.302-3.696; $\mathrm{P}=0.003$ ) and intraoperative radiofrequency ablation (HR: 2.291; 95\% CI: 1.081-4.855; $\mathrm{P}=0.031$ ) were significant negative predictors of 5-year OS. Multivariate analysis showed that WNT6 expression (HR: 2.089; 95\% CI: 1.231-3.545; $\mathrm{P}=0.006)$, $\mathrm{N}$ stage (HR: 2.131; 95\% CI: 1.132-4.015; $\mathrm{P}=$ 0.019 ), number of liver metastatic tumors (HR: 2.318; 95\% CI: 1.220-4.405; $\mathrm{P}=0.010)$ and preoperative CA19-9 level (HR: 1.931; 95\% CI: 1.135-3.286; P = $0.015)$ were identified as independent predictors of 5-year OS.

B

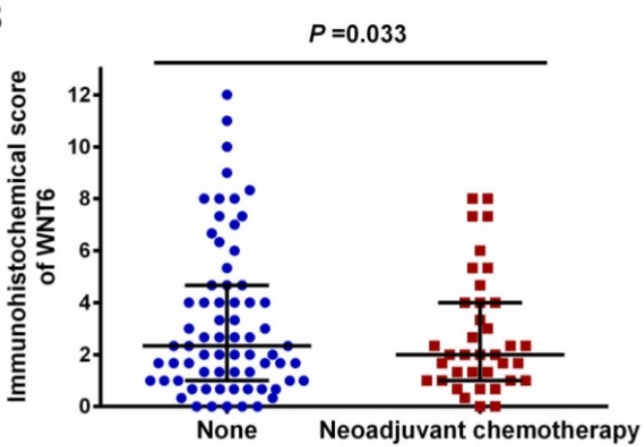

Figure 3. Expression level of WNT6 in different clinical risk scores (CRS) patients and patients with or without preoperative chemotherapy. (A) The expression level of WNT6 was comparable in different CRS patients $(P=0.995)$. (B) The expression level of WNT6 was significantly lower in patients with preoperative chemotherapy than in those without preoperative chemotherapy $(P=0.003)$.

A

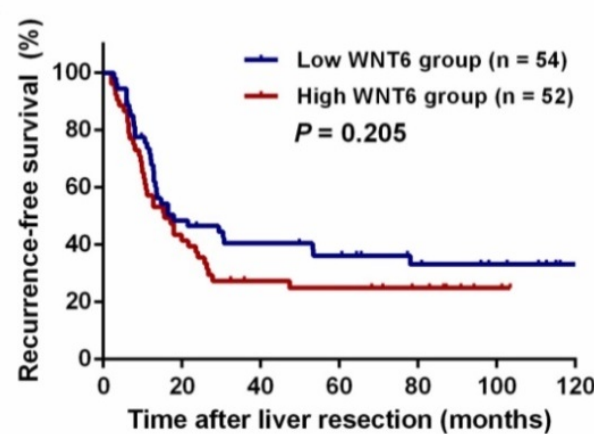

B

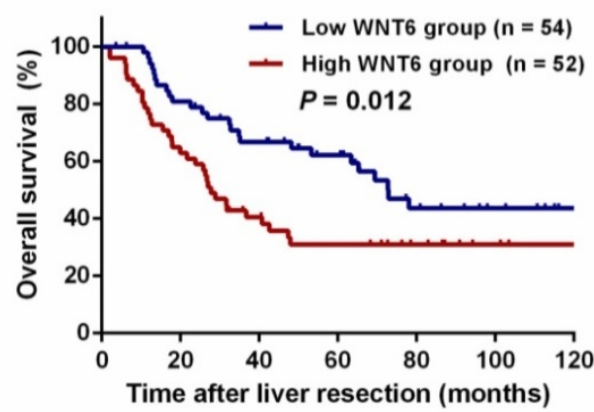

Figure 4. Kaplan-Meier long-term survival curves grouped by high and low WNT6 expression in CRLM patients. (A) Recurrence-free survival (RFS) in all patients. (B) Overall survival (OS) in all patients.

A

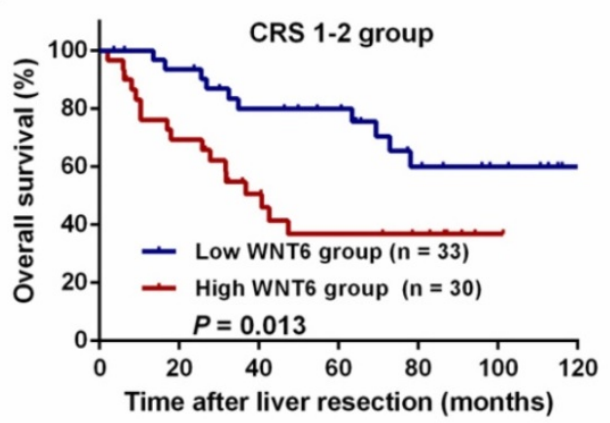

B

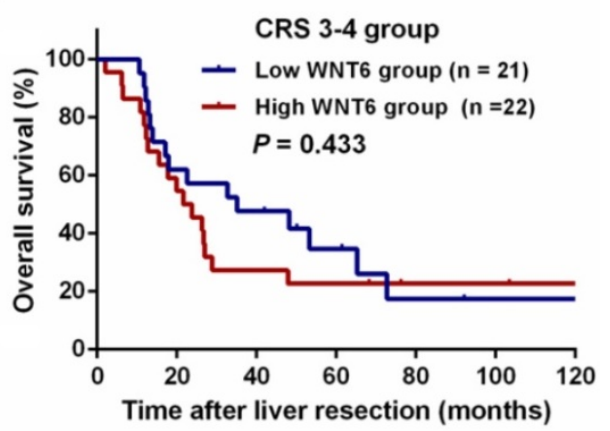

Figure 5. Kaplan-Meier curves for the comparison of long-term survival rates based on different WNT6 expression in high- and low-risk patients. (A) OS of patients with low-risk CRLM. (B) OS of patients with high-risk CRLM. 
Table 3. Univariate and multivariate Cox regression analysis of overall survival in patients with colorectal liver metastasis after liver resection

\begin{tabular}{|c|c|c|c|c|}
\hline & Univariate & & Multivariate & \\
\hline Variable & HR $(95 \%$ CI $)$ & P value & HR $(95 \%$ CI $)$ & $\mathrm{P}$ value \\
\hline Age ( $\leq 60$ year vs. $>60$ year) & $1.004(0.597-1.689)$ & 0.987 & & \\
\hline Sex (Male vs. Female) & $1.140(0.660-1.969)$ & 0.638 & & \\
\hline Primary tumor location (Rectum vs. Colon) & $1.145(0.682-1.921)$ & 0.609 & & \\
\hline Primary tumor differentiation (Poor vs. Well to moderate) & $1.097(0.520-2.316)$ & 0.807 & & \\
\hline T stage (4 vs. $1-3)$ & $1.256(0.741-2.131)$ & 0.397 & & \\
\hline $\mathrm{N}$ stage (1-2 vs. 0$)$ & $1.922(1.034-3.572)$ & 0.039 & $2.131(1.132-4.015)$ & 0.019 \\
\hline Number of liver metastatic tumors ( $>3$ vs. $1-3$ ) & $2.481(1.320-4.663)$ & 0.005 & $2.318(1.220-4.405)$ & 0.010 \\
\hline Metastases diameter ( $>3 \mathrm{~cm}$ vs. $\leq 3 \mathrm{~cm})$ & $1.462(0.872-2.453)$ & 0.150 & & \\
\hline Preoperative CEA (>10 ng/ml vs. $\leq 10 \mathrm{ng} / \mathrm{ml}$ ) & $1.688(0.999-2.851)$ & 0.050 & & \\
\hline Preoperative CA19-9 (>35 U/ml vs. $\leq 35 \mathrm{U} / \mathrm{ml}$ ) & $2.194(1.302-3.696)$ & 0.003 & $1.931(1.135-3.286)$ & 0.015 \\
\hline Intraoperative radiofrequency ablation (Yes vs. No) & $2.291(1.081-4.855)$ & 0.031 & $1.773(0.728-4.320)$ & 0.208 \\
\hline Preoperative chemotherapy (Yes vs. No) & $1.065(0.604-1.878)$ & 0.826 & & \\
\hline Adjuvant chemotherapy (Yes vs. No) & $0.857(0.389-1.888)$ & 0.702 & & \\
\hline WNT6 expression (High vs. Low) & $1.920(1.143-3.225)$ & 0.014 & $2.089(1.231-3.545)$ & 0.006 \\
\hline
\end{tabular}

Abbreviations: CEA: carcinoembryonic antigen, CA19-9: carbohydrate antigen 19-9

\section{Discussion}

To further understand tumorigenesis and explore significant biomarkers to predict long-term survival in CRLM, the present study evaluated WNT6 expression in tumor tissue of CRLM patients for the first time. Our data show that WNT6 is commonly expressed in colon cancer tumor tissues. Furthermore, overexpression of WNT6 in tumor tissue was correlated with poor OS in CRLM patients, especially in those with low-risk CRLM. Our results also indicate that WNT6 expression was an independent prognostic factor for 5-year OS. These results implied that WNT6 might be used as a potential prognostic biomarker for CRLM.

Several molecular mechanisms could contribute to the reduced long-term survival observed with high WNT6 expression in CRLM patients. WNT6 promotes tumorigenesis and progression of colon cancer [23], and overexpression of WNT6 may increase the expression of caspase-3 and MMP2. It is well known that MMP2 is involved in the proteolytic degradation of the extracellular matrix, which subsequently promotes the epithelial to mesenchymal transition (EMT), a key process involved in cancer metastasis[28, 29]. Moreover, WNT6 was also confirmed to be an important component of EMT initiation [30]. These findings suggest that WNT6 plays an important role in maintaining metastatic aggressiveness in CRC cells. Chemotherapy resistance is another factor that compromises the prognosis of CRLM patients. Our data showed that patients receiving preoperative chemotherapy presented significantly lower WNT6 expression than those without preoperative chemotherapy $(\mathrm{P}=0.033)$. We hypothesized that chemotherapy decreases the expression level of WNT6 in CRLM and that the effect of chemotherapy might be associated with WNT6 expression. If high expression of WNT6 after chemotherapy was detected, the effect of chemotherapy might be not approving. A previous study indicated that WNT6 was able to facilitate the development of resistance to chemotherapeutic drugs [21,31]. The increased WNT6 expression rendered tumor cells resistant to apoptosis, thus favoring tumor cell survival and further progression [21]. In addition, enhancing the expression of WNT6 increases the cisplatin resistance of bladder cancer cells [31]. Accordingly, we considered WNT6 to be a potential target to overcome chemoresistance in cancer patients.

Although WNT6 expression was not significantly associated with CRS, we found that WNT6 expression was associated with OS in low-risk patients but not in high-risk patients. We considered this to be mainly attributed to the disparate characteristics between different-risk CRLM patients. CRLM patients with CRS 0-2 have a highly favorable outcome, while patients with a CRS of 3-5 have a much more guarded prognosis [27, 32]. As clinical risk factors accumulate, their effect on prognosis increases.

In addition, Ayez $\mathrm{N}$ et al. study has demonstrated that traditional CRS systems including Fong system are not a reliable prognostic tool when used in patients before treatment with preoperative chemotherapy except for the Nordlinger CRS system [33]. In fact, there were $29.2 \%$ patients were performed preoperative chemotherapy in the current study, which might affect the prognostic analysis value of Fong CRS system. Therefore, the prognostic predictive effect of WNT6 expression is more difficult to discern. This finding suggested that the long-term survival of low-risk patients might be more susceptible to WNT6 expression.

Based on the result of our study, the feasibility of WNT6 expression could be used to categorize CRLM patients as a stratification parameter for different prognosis. In addition, the detection of WNT6 expression could also help clinicians tailor adjuvant 
treatment in a comprehensive transversal approach. Accordingly, if patients were identified as high WNT6 expression, they might be benefit from a more aggressive postoperative chemotherapy, even targeted therapy and a more normative follow-up measurement. Otherwise, patients with low WNT6 expression tumors, they might have less benefit from postoperative chemotherapy as they presented a favorable prognosis. Accordingly, the enhanced postoperative chemotherapy should be avoided. Therefore, they can be spared the associated toxicity, cost, and inconvenience of over-treatment. Taken together, detection of WNT6 expression might be useful to personalizing treatment which allows patients to maximize benefits while minimizing harms, thus providing optimal survival benefit and quality of life.

Some limitations of this study should be acknowledged. First, this retrospective study was conducted with an uncontrolled methodology and included a limited number of patients from a single center. Therefore, the findings need to be validated in multicenter studies or larger cohort studies. Second, liver metastatic tumors were not included in this study to detect WNT6 expression. This limitation may have led to an underestimation of the prognostic impact of WNT6 on the long-term survival of CRLM patients. Moreover, molecular features of tumors, such as microsatellite instability (MSI), CpG island methylator phenotype (CIMP) level, and BRAF and KRAS mutational status, were not evaluated in this study; an analysis of the association of WNT6 with these molecular markers would help us further understand the impact of WNT6 on tumorigenesis in CRLM. Future studies should examine these biomarkers. Despite these limitations, our findings suggest that WNT6 expression should be considered a routine postoperative measurement for CRLM patients undergoing liver resection.

In conclusion, this study demonstrated that WNT6 expression is a valuable predictor of OS in patients with CRLM undergoing hepatic resection. Herein, the detection of WNT6 expression may help surgeons evaluate the benefit of curative hepatectomy and formulate individualized strategies for postoperative treatment.

\section{Acknowledgements}

We deeply appreciate the help from all colleagues of the colorectal surgery department at Sun Yat-sen University Cancer Center, who have worked in obtaining the tissue specimens and provided it to the current study. The correspondence authors, on behalf of all coauthors, take full responsibility for the analyses in this study. The authenticity of this article has been validated by uploading the key raw data onto the Research Data Deposit public platform (www.researchdata.org.cn), with the approval number as RDDA2018000347.

\section{Ethics approval and consent to participate}

Study was approved by the Institutional Research Ethics Committee of Sun Yat-sen University Cancer Center (Approval number: GZR2017-004). The informed consents for using blood samples, before the initial treatment, were obtained from the patients.

\section{Availability of data and materials}

The datasets analysed during the current study were available from the corresponding author on reasonable request. Anyone who is interested in the information should contact zhanglin@sysucc.org.cn.

\section{Funding}

This study was funded by the Natural Science Foundation of Guangdong Province, China (grant no. 2018A030310260), National Natural Science Foundation of China (NO. 81772595), and Sun Yat-sen University Clinical Research 5010 Program (2015024).

\section{Competing Interests}

The authors have declared that no competing interest exists.

\section{References}

1. Chen W, Zheng R, Baade P, Zhang S, Zeng H, Bray F, et al. Cancer statistics in China, 2015. CA Cancer J Clin. 2016;66:115-32.

2. Allemani C, Matsuda T, Di Carlo V, Harewood R, Matz M, Nikšić M, et al. Global surveillance of trends in cancer survival 2000-14 (CONCORD-3): analysis of individual records for 37513025 patients diagnosed with one of 18 cancers from 322 population-based registries in 71 countries. Lancet. 2018;391:1023-75.

3. O'Reilly D, Poston G. Colorectal liver metastases: current and future perspectives. Future Oncol. 2006;2:525-31.

4. Van Cutsem E, Nordlinger B, Adam R, Köhne C, Pozzo C, Poston G, et al. Towards a pan-European consensus on the treatment of patients with colorectal liver metastases. Eur J Cancer. 2006;42:2212-21.

5. Wong R, Cunningham D, Barbachano Y, Saffery C, Valle J, Hickish T, et al. A multicentre study of capecitabine, oxaliplatin plus bevacizumab as perioperative treatment of patients with poor-risk colorectal liver-only metastases not selected for upfront resection. Ann Oncol. 2011;22:2042-8.

6. Ye L, Liu T, Ren L, Wei Y, Zhu D, Zai S, et al. Randomized controlled trial of cetuximab plus chemotherapy for patients with KRAS wild-type unresectable colorectal liver-limited metastases. J Clin Oncol. 2013;31:1931-8.

7. Akgül Ö, Çetinkaya E, Ersöz Ş, Tez M. Role of surgery in colorectal cancer liver metastases. World J Gastroenterol. 2014;20:6113-22.

8. D'Angelica M, Kornprat P, Gonen M, DeMatteo R, Fong Y, Blumgart L, et al. Effect on outcome of recurrence patterns after hepatectomy for colorectal metastases. Ann Surg Oncol. 2011;18:1096-103.

9. Tabchouri N, Gayet B, Okumura S, Donatelli G, Beaussier M, Bennamoun M, et al. Recurrence patterns after laparoscopic resection of colorectal liver metastases. Surg Endosc. 2018; doi: 10.1007/s00464-018-6229-6. [Epub ahead of print].

10. Lin J, Peng J, Zhao Y, Luo B, Zhao Y, Deng Y, et al. Early recurrence in patients undergoing curative resection of colorectal liver oligometastases: identification of its clinical characteristics, risk factors, and prognosis. J Cancer Res Clin Oncol. 2018;144:359-69.

11. Imai K, Allard M-A, Benitez CC, Vibert E, Sa Cunha A, Cherqui D, et al. Early Recurrence After Hepatectomy for Colorectal Liver Metastases: What Optimal Definition and What Predictive Factors? The oncologist. 2016;21:887-94.

12. Brudvik K, Jones R, Giuliante F, Shindoh J, Passot G, Chung M, et al. RAS Mutation Clinical Risk Score to Predict Survival After Resection of Colorectal Liver Metastases. Ann Surg. 2017; doi: 10.1097/SLA.0000000000002319. [Epub ahead of print]. 
13. Rees M, Tekkis P, Welsh F, O'Rourke T, John T. Evaluation of long-term survival after hepatic resection for metastatic colorectal cancer: a multifactorial model of 929 patients. Ann Surg. 2008;247:125-35.

14. Stewart D. Wnt signaling pathway in non-small cell lung cancer. J Natl Cancer Inst. 2014;10:djt356.

15. Tai D, Wells K, Arcaroli J, Vanderbilt C, Aisner DL, Messersmith WA, et al. Targeting the WNT Signaling Pathway in Cancer Therapeutics. The oncologist. 2015;20:1189-98.

16. Peifer M, Polakis P. Wnt signaling in oncogenesis and embryogenesis--a look outside the nucleus. Science. 2000;287:1606-9.

17. Jiang Z, Pan L, Chen X, Chen Z, Xu D. Wnt6 influences the viability of mouse embryonic palatal mesenchymal cells via the $\beta$-catenin pathway. Experimental and Therapeutic Medicine. 2017;14:5339-44.

18. Schaale K, Brandenburg J, Kispert A, Leitges M, Ehlers S, Reiling N. Wnt6 is expressed in granulomatous lesions of Mycobacterium tuberculosis-infected mice and is involved in macrophage differentiation and proliferation. J Immunol. 2013;191:5182-95.

19. Wang Q, Lu J, Zhang S, Wang S, Wang W, Wang B, et al. Wnt6 is essential for stromal cell proliferation during decidualization in mice. Biol Reprod. 2013;88:5.

20. Galbraith R, Poole E, Duggan D, Muehling J, Hsu L, Makar K, et al. Polymorphisms in WNT6 and WNT10A and colorectal adenoma risk. Nutr Cancer. 2011;63:558-64.

21. Yuan G, Regel I, Lian F, Friedrich T, Hitkova I, Hofheinz R, et al. WNT6 is a novel target gene of caveolin-1 promoting chemoresistance to epirubicin in human gastric cancer cells. Oncogene. 2013;32:375-87.

22. Zhang L, Yuan G, Fang Y, Qiu M, Lin J, Sun J, et al. Increased WNT6 expression in tumor cells predicts unfavorable survival in esophageal squamous cell carcinoma patients. International journal of clinical and experimental pathology. 2015;8:11421-7.

23. Zheng $\mathrm{X}-\mathrm{L}, \mathrm{Yu} \mathrm{H}-\mathrm{G}$. Wnt6 contributes tumorigenesis and development of colon cancer via its effects on cell proliferation, apoptosis, cell-cycle and migration. Oncology Letters. 2018;16:1163-72.

24. Peng $\mathrm{J}, \mathrm{Li} \mathrm{H}, \mathrm{Ou} \mathrm{O}$, Lin $\mathrm{J}$, Wu $\mathrm{X}, \mathrm{Lu} \mathrm{Z}$, et al. Preoperative lymphocyteto-monocyte ratio represents a superior predictor compared with neutrophil-to-lymphocyte and platelet-to-lymphocyte ratios for colorectal liver-only metastases survival. Onco Targets Ther. 2017;10:3789-3799.

25. Peng $\mathrm{I}, \mathrm{Ou} \mathrm{O}, \mathrm{Wu} X$, Zhang $\mathrm{R}$, Zhao $\mathrm{O}$, Jiang $\mathrm{W}$, et al. Expression of voltage-gated sodium channel Nav1.5 in non-metastatic colon cancer and its associations with estrogen receptor (ER)- $\beta$ expression and clinical outcomes. Chin J Cancer. 2017;36:89.

26. Peng J, Ou Q, Guo J, Pan Z, Zhang R, Wu X, et al. Expression of a novel CNPY2 isoform in colorectal cancer and its association with oncologic prognosis. Aging (Albany NY). 2017;9:2334-51.

27. Fong Y, Fortner J, Sun RL, Brennan MF, Blumgart LH. Clinical score for predicting recurrence after hepatic resection for metastatic colorectal cancer: analysis of 1001 consecutive cases. Annals of surgery. 1999;230:309-18; discussion 18-21.

28. Gialeli C, Theocharis A, Karamanos N. Roles of matrix metalloproteinases in cancer progression and their pharmacological targeting. FEBS J. 2011;278:16-27.

29. Kalluri R, Weinberg RA. The basics of epithelial-mesenchymal transition. The Journal of clinical investigation. 2009;119:1420-8.

30. Krawetz R, Kelly G. Coordinate Galpha13 and Wnt6-beta-catenin signaling in F9 embryonal carcinoma cells is required for primitive endoderm differentiation. Biochem Cell Biol. 2009;87:567-80

31. Fan $Y$, Shen B, Tan M, Mu X, Qin Y, Zhang F, et al. Long non-coding RNA UCA1 increases chemoresistance of bladder cancer cells by regulating Wnt signaling. The FEBS journal. 2014;281:1750-8.

32. Pan Z, Peng J, Lin J, Chen G, Wu X, Lu Z, et al. Is there a survival benefit from adjuvant chemotherapy for patients with liver oligometastases from colorectal cancer after curative resection? Cancer Communications. 2018;38:29.

33. Ayez N, Lalmahomed ZS, van der Pool AE, Vergouwe Y, van Montfort K, de Jonge $\mathrm{J}$, et al. Is the clinical risk score for patients with colorectal liver metastases still useable in the era of effective neoadjuvant chemotherapy? Ann Surg Oncol. 2011;18:2757-63. 